\title{
Implementation of a Z-matrix approach within the SIESTA periodic boundary conditions code and its application to surface adsorption
}

\author{
R. C. HOFT ${ }^{1}$, J. D. GALE ${ }^{2}$ and M. J. FORD ${ }^{1 *}$ \\ ${ }^{1}$ Institute for Nanoscale Technology, University of Technology Sydney, PO Box 123, \\ Broadway NSW 2007, Australia \\ ${ }^{2}$ Nanochemistry Research Institute, Curtin University of Technology, PO Box U1987, \\ Perth, WA 6845, Australia
}

(Received )

We implement a flexible Z-matrix approach in the Density Functional Theory (DFT) periodic boundary conditions code, SIESTA. This allows a mixture of Z-matrix and Cartesian coordinates to be used for geometry specification and optimisation. In addition, geometry constraints in the form of fixed coordinates and fixed linear relationships between coordinates can be specified. A Z-matrix approach in condensed phase calculations can be advantageous, for example in studying molecular adsorption onto a surface, both in terms of flexibility and efficiency. We demonstrate our implementation for the case of thiol adsorption on the $\mathrm{Au}(111)$ surface.

PACS numbers: 68.43.Bc; 82.20.Wt; 89.20 Ff

\section{Introduction}

Corresponding author email: mike.ford@uts.edu.au 
Geometry optimisation is a very important aspect of electronic structure calculations, as a pre-requisite to property determination, and typically is the most time-consuming part of any study. Consequently, various ways to improve performance have been investigated through trying to reduce the number of steps required for convergence. This is predicated on the assumption that the system initial lies within the basin of the relevant local minimum, since for complex materials it is impossible to ensure that the state of globally lowest energy has been identified. In addition to this, it is often desirable to be able to find transition states as well as minima.

Important considerations for efficient optimisation include the optimisation algorithm, the initial guess and updating method of the Hessian, the initial geometry and the coordinate system used. Three main types of coordinate systems have been used extensively in the literature: Cartesians, redundant internals and non-redundant internals. Cartesian coordinates perform well for cyclic molecules where non-redundant coordinates are troublesome due to coupling between coordinates [1, 2]. However, non-redundant internals (for example Z-matrix internal coordinates) can be preferable in acyclic molecules and it has been suggested that mixed Cartesian and non-redundant internal coordinates be used to combine the best of both approaches [1]. Different sets of redundant internal coordinates have been constructed [3-5]. These generally perform better for optimising molecules especially for flexible and polycyclic molecules.

Internal coordinates are a more intuitive way to specify molecular structure, where the molecule is specified in terms of bond lengths, angles and torsions. As well as efficiency gains, due to the ability to better precondition the algorithm given the nature of 
the force constants involved, internal coordinates provide greater flexibility in, for example, partial or constrained optimisations. Indeed, most, if not all, ab initio electronic structure codes for molecules contain some implementation of internal coordinates, Zmatrix and/or redundant internals. Implementation of internal coordinates for periodic boundary conditions (PBC) codes, by contrast is rare. However, the same advantages can be gained in this case as for molecules, both in terms of flexibility and efficiency, particularly for molecular adsorption onto a surface.

In the present work we have extended the capabilities of the widely used SIESTA code $[6,7]$, by allowing the user to input the coordinates in the Z-matrix non-redundant internal coordinate format. Until recently atomic coordinates were always input in Cartesian or fractional (scaled) format and geometry optimisations performed using the conjugate gradients (CG) algorithm on these globally referenced coordinates. In this paper we describe our combined Z-matrix periodic boundary conditions implementation and give examples of its application to structure optimisation for isolated molecules and molecular adsorption onto surfaces.

\section{Methods}

All the calculations in this paper were conducted with the SIESTA software package. This program implements the SIESTA methodology for linear-scaling density functional theory within periodic boundary conditions and is based on the linear combination of atomic orbitals approximation. The valence electrons are described by atom-centered basis sets, and the nucleus/core electrons are represented by norm-conserving 
pseudopotentials. The key feature of this methodology is that the orbitals are strictly localized in real-space, with a cut-off radius defined by a single energy shift parameter for all atoms that represents the energy increase in the orbitals due to confinement. Exploitation of this locality leads directly to linear-scaling without the requirement of neglecting integrals based on a threshold value.

The code has been modified to allow coordinate input in a mixed Cartesian internal coordinate format, which is particularly convenient for studying molecules on surfaces. The internal coordinates are specified as in the usual Z-matrix format by a bond length, bond angle and dihedral. The first atom in the Z-matrix is specified in Cartesian coordinates, or fractional coordinates of the unit cell in order to determine the absolute location of the molecular fragment. For the second atom, the bond length is given relative to the first atom and then it's remaining degrees of freedom are specified by the angle between the interatomic vector from atom 1 to 2 and the z-axis, as well as the dihedral angle between this interatomic vector and the $\mathrm{x}$-axis, as viewed down the z-axis. This definition is equivalent to placing a dummy atom along the z-axis, followed by one along the x-axis. For the third atom, the bond length and angle can be defined in terms of the first two atoms, with the dihedral being relative to the first two atoms and z-axis. For remaining atoms, the freedom exists to define the atom in terms of any of the foregoing species. As is always the case in Z-matrix geometry specification, one has to take care to avoid a set of internal coordinates that are co-linear.

A separate block can be specified in addition to the Z-matrix in order to define atoms in Cartesian or fractional space. Apart from building the initial geometry, the new input format is designed to offer greater control over the optimisation process through 
specifying which degrees of freedom are to be included in the process. Geometry optimisation flags can be set for every coordinate such that the coordinate varies, is fixed, or is constrained to be a linear function of another coordinate during the optimisation. Furthermore, symbolic variables can be included in the Z-matrix to facility the control of molecule symmetry by equivalencing of geometric parameters.

Geometry optimisation is performed in internal coordinates for the Z-matrix portion of system, and convergence criteria specified separately for forces relating to bond-length and angle. This implementation of internal coordinates provides ease and flexibility when setting up geometries that are simply not available in most PBC codes. Molecules can be docked and manipulated on surfaces very easily. Flexibility of the optimisation is useful for restricted optimisations, such as locating transition states or scanning potential surfaces, as required in the present study.

To illustrate the application of the mixed Z-matrix / Cartesian optimiser, we present results for the adsorption of thiols on the surface of gold. For these calculations the generalized-gradient approximation (GGA) to the exchange-correlation functional due to Perdew, Burke and Ernzerhof (PBE) [8] is used throughout. All calculations are spin restricted and employ a Fermi smearing of $25 \mathrm{meV}$ to aid convergence. Norm-conserving pseudopotentials (with a relativistic correction in the case of $\mathrm{Au}$ ) generated according to the scheme of Troullier and Martins [9] are used. A double-zeta plus single polarisation function is used to represent the 1 s electrons in $\mathrm{H}, 2 \mathrm{~s}$ and $2 \mathrm{p}$ electrons in $\mathrm{C}$ and $6 \mathrm{~s}$ and $5 \mathrm{~d}$ electrons in Au. Transferability of the pseudopotentials has been previously tested by comparison with well-known experimental results and all-electron calculations [10]. 
For the isolated molecules, an energy shift of 5 mRy was used in the confinement of the basis set and an effective kinetic energy cut-off for the real space integration grid of 200 Ry was employed. Only a single k-point in reciprocal space is required for the determination of the isolated molecules. Each molecule is placed in a cubic unit cell of 20 $\AA$, as this was found to be sufficient to lead to negligible intermolecular interactions. The computational conditions used here are reasonably high and give relatively wellconverged relative energies. Three sets of increasingly strict force tolerances were used for lengths and angles: I (0.04 eV/Å; $0.0009 \mathrm{eV} / \mathrm{deg})$, II (0.02 eV/Å;0.0004 eV/deg) and III $(0.01 \mathrm{eV} / \AA \AA$; $0.0002 \mathrm{eV} / \mathrm{deg})$ The maximum displacements were increased from the default values to $0.4 \AA$ and 2 degrees.

For the calculations of thiol adsorption on $\mathrm{Au}(111)$ the gold substrate was modeled by a 3 layer slab with a 3x3 unit cell. For methanethiol adsorption this is quite adequate to ensure no molecule-molecule interaction on the surface. For benzenethiol it is about the minimum tolerable cell size. For the present work, the aim is not to determine precise binding energies for the low coverage limit, but rather to demonstrate the utility of the Z-matrix approach. However, a relatively high level of computational conditions have been used that give total energies converged to better than $5 \mathrm{kcal} / \mathrm{mol}$ [10] with respect to numerical integration factors, as opposed to the basis set expansion. For the surface calculations, a $3 \times 3 \times 1 \mathrm{k}$-point grid is used in reciprocal space. This relatively sparse grid is used in the interest of computational time and provides adequate results compared with denser grids [10]. As in the isolated molecule calculations, a $5 \mathrm{mRy}$ energy shift and a 200 Ry effective plane-wave cut-off are used. The force tolerance was 
set at level I (0.04 eV/A for lengths and $0.0009 \mathrm{eV} / \mathrm{deg}$ for angles). Maximum displacements are also identical to the isolated molecule case.

\section{Results and Discussion}

In order to assess the utility of our Z-matrix implementation, we have performed geometry optimisations for the following representative molecules in isolation, water, benzene and hexanedithiol, and for two typical molecular adsorption problems, methanethiol on $\mathrm{Au}(111)$ and benzenethiol on $\mathrm{Au}(111)$. The intent is to compare optimisations performed in Cartesian coordinates and internal coordinates to assess optimisation efficiency, flexibility and robustness.

\subsection{Isolated Molecules}

The number of conjugate gradient steps with three force tolerance levels needed to optimise the isolated molecules using Cartesian and internal coordinates are given in Table 1. The starting geometries are identical in each coordinate system and represent a reasonable guess at the optimum geometry for all molecules. In addition, Table 1 presents the number of steps needed to converge the optimisation as a function of different numbers of optimisation variables. The number of internal degrees of freedom for an isolated molecule with $N$ atoms is $3 N-6$. However, in the Cartesian implementation the only constraints supported are fixing the coordinates of an atom. Thus to relax all the internal degrees of freedom $3 N-3$ variables are utilised by fixing the coordinates of one 
atom. In the Z-matrix implementation, any of the optimisation variables can be fixed. Thus for a completely unconstrained optimisation, only $3 N-6$ variables are needed. This is achieved by fixing the Cartesian coordinates of the first atom in the Z-matrix description as well as the three external rotational degrees of freedom, being the second and third coordinates of the second atom together with the third coordinate of the third atom. However, symmetry arguments may lead to a reduction of variables, e.g. the O-H bonds in water are equal and thus the optimisation can be done with only two variables. In benzene, the $\mathrm{C}-\mathrm{C}$ bonds in the ring are identical, as are the $\mathrm{C}-\mathrm{H}$ bonds. Further assuming that the molecule is flat reduces the number of variables to two.

[insert table 1 about here]

Clearly the Z-matrix performs much better in the simple case of water. However, the use of symmetry in the Z-matrix optimisation does not improve the performance any further. In the chain-like hexanedithiol, again the Z-matrix optimisation converges in fewer steps at the level I force tolerance. At stricter tolerances the Z-matrix performs well when the external degrees of freedom are fixed, but optimisation becomes troublesome when these are relaxed. Fixing one of the atoms in the Cartesian optimisation significantly increases the number of optimisation steps. In the case of the benzene molecule, the Cartesian coordinate optimisation performs better than the Z-matrix. As discussed earlier, this is the expected behaviour for cyclic molecules [1, 2]. Here the efficiency is improved drastically by using the symmetry of the molecule to reduce the number of variables in the Z-matrix case. This is straight forward in the Z-matrix implementation, but difficult to implement in Cartesian coordinates, and is not currently available in SIESTA. Again fixing one of the atoms increases the number of optimisation 
steps in Cartesian coordinates. Because there is no Hessian, the conjugate gradient method is not sensitive to the translational invariance problem that would cause this matrix to be uninvertable unless a coordinate is fixed. Unless the forces acting on the variable atoms are modified to allow for those of the fixed atom being effectively shifted to zero, it is not surprising that the performance of the conjugate gradients algorithm deteriorates when the whole system is not relaxed in Cartesian space.

The final geometries of the molecules are not sensitive to either the number of optimisation variables or the force tolerance level. The maximum geometry variation is about $0.002 \AA$ for bond lengths and $1.5^{\circ}$ for angles.

\subsection{Surface Adsorption}

The systems for the molecular adsorption optimisation problems studied are shown in Figure 1. In both cases the terminal hydrogen has been removed from the sulphur atom, which is then bonded directly to the surface. The sulphur then forms a relatively strong covalent bond with the surface [11]. There are also reports that a weaker bond can form with the hydrogen atom intact [12] (we do not consider this case). The optimum geometry for methanethiol is well known and is with the sulphur atom adsorbed in the bridge site slightly displaced towards the fcc hollow with the methane tail group tilted at an angle of about $50^{\circ}$ to the surface normal over the bridge site [12]. For benzenethiol, both the bridge and bridge-fcc sites are minima, again with the benzene tail group tilted at $68^{\circ}$ and $56^{\circ}$ with respect to the surface normal [13].

[insert figure 1 about here] 
We have performed geometry optimisations for the two adsorbates using the same starting geometry for both Cartesian and internal coordinates. In all cases, the gold slab atom positions are described by Cartesian coordinates. The adsorbate molecules are initially in the bridge site and perpendicular to the surface. A very small tilt angle is required to avoid poorly specified coordinates for the Z-matrix. This can be avoided by orienting the slab such that the surface normal is the x-axis, rather than the more conventional z-axis. In any case, this has no effect upon the results presented. The gold atoms are fixed in their bulk positions, i.e. no surface relaxation is allowed. This has a relatively small effect on the final geometries, and would increase the number of optimisation steps if included for both Cartesian and internal coordinates by roughly the same number. In Cartesian coordinates the entire molecule is allowed to relax, included the sulphur position. For the Z-matrix input geometry a single optimisation where all adsorbate coordinates are free, and a two-step optimisation where initially the sulphur atom position and attitude of molecule are relaxed followed by relaxation of the entire adsorbate were compared.

Cartesian coordinate optimisations for methanethiol and benzenethiol converged in a very small number of steps, namely 3 and 4, respectively. However, the final geometry is not the known minimum. In both cases the optimised geometry found here was with the molecule remaining in the bridge site and upright on the surface. Even starting from a geometry where the methanethiol adsorbate is displaced slightly ( $0.3 \AA$ ) towards the fcc hollow results in a final geometry where the sulphur atom has moved back to the bridge site. Increasing the force convergence tolerance by a factor of 4 increases the number of 
optimisation steps to 31 and 91 for methanethiol and benzenethiol respectively, but still gives an upright molecule adsorbed in the bridge site.

The single step methanethiol optimisation using internal coordinates converged to the correct geometry in 60 steps. The sulphur atom is displaced $0.27 \AA$ towards the hollow site and the tail is tilted back across the bridge site at $56^{\circ}$. Performing the optimisation in two stages reduces the total number of steps slightly to 53, and gives the same final geometry. For such a small molecule it is not surprising that the two methods relax in about the same number of steps. Increasing the maximum angle step size may result in more efficient optimisation. However, it is clear from the above results that internal coordinate optimisation is considerably more robust in terms of finding the global minimum compared with Cartesian coordinates.

Internal coordinate optimisation of benzenethiol in a single step converged in 204 steps, and again reached the correct geometry with the sulphur atom located in the bridge site and tilted at $68^{\circ}$ to the surface normal. A two-stage optimisation in this case is significantly more efficient taking only 58 steps to find the same minimum. This example also demonstrates the robustness of internal coordinate optimisation for surface adsorption. The advantage lies in the fact that the potential energy surface (PES) can be relatively flat with respect to tilting of the molecule on the surface. Internal coordinates allow the angle optimisation to be decoupled from the bond-length optimisation and different tolerances to be specified for each, whereas in Cartesian coordinates this cannot be easily achieved. Hence, internal coordinate optimisations explore this flat potential energy surface much more efficiently.

[insert figure 2 about here] 
As a final example, Figure 2 shows a scan of the PES for the methanethiol adsorbate. Here the sulphur atom is scanned along the atop-hcp-bridge-fcc-atop line, and at each point in the scan a constrained optimisation is performed where the $\mathrm{S}$ atom is fixed laterally but relaxes its height above the surface and the remainder of the molecule relaxes. The potential well along this reaction coordinate is approximately $0.37 \mathrm{eV}$ deep, with no barrier apparent at the bridge site. Previous calculations scanning a rigid molecule across the surface have predicted a barrier of $0.4 \mathrm{eV}$ at the bridge site [14].

The two hollow sites are local maxima and not minima, at least along this reaction coordinate. We have tried a Cartesian optimisation starting at these two sites, with the adsorbate normal to the surface, which converge to an apparent minimum with the adsorbate remaining perpendicular.

Scanning this PES is extremely time-consuming to achieve in Cartesian coordinates. The usual approach to scanning PES's for molecular adsorption is a brute force scan of a rigid molecule in different configurations across the surface many times, with different attitudes and heights. Here the same outcome has been achieved in a single scan. This has obvious extension to the identification of transition states.

\section{Conclusions}

An internal coordinates geometry specification and optimisation has been implemented in the first principles quantum chemical code, SIESTA. This code uses periodic boundary conditions and can therefore be applied to such problems as surface adsorption. A mixed input geometry is allowed where the substrate can be specified in conventional Cartesian 
coordinates, while the adsorbate can be specified in a Z-matrix format. Geometry optimisation is then carried out in this mix of Cartesian - internal coordinates.

This approach provides greater flexibility for setting up substrate-adsorbate geometries and for performing constrained optimisations, potential energy scans and transition state searches. In certain circumstances optimisations can be performed more efficiently by allowing a series partial relaxations, and independent optimisation of angles and bond-lengths can be a more robust method for finding global minima.

The present work has been performed using the existing conjugate gradient optimiser within SIESTA. However, the natural extension to a Newton-Raphson algorithm based on BFGS updating of an initial Hessian is likely to bring significant performance benefits when working in internal coordinates due to the ability to precondition each mode with reasonable force constants based on whether the coordinate represents a bond, angle, torsion or intermolecular force. Consequently, even greater benefits in efficiency, as well as flexibility, can be expected in future.

\section{Acknowledgements}

This work was supported by the Australian Research Council. Computational resources were provided under the merit allocation schemes of ac3 (in NSW) and the National Facility, APAC. JDG would like to thank the Government of Western Australia for support under the Premier’s Research Fellowship Program.

\section{References}


[1] H. B. Schlegel. A comparison of geometry optimization with internal, cartesian, and mixed coordinates. Int. J. Quantum Chem., 44, 243 (1992).

[2] J. Baker and W. J. Hehre. Geometry optimization in cartesian coordinates - the end of the z-matrix. J. Comp. Chem., 12, 606 (1991).

[3] P. Pulay and G. Fogarasi. Geometry optimization in redundant internal coordinates. J. Chem. Phys., 96, 2856 (1992).

[4] C. Y. Peng, P. Y. Ayala, H. B. Schlegel and M. J. Frisch. Using redundant internal coordinates to optimize equilibrium geometries and transition states. J. Comp. Chem., 17, 49 (1996).

[5] G. Fogarasi, X. F. Zhou, P. W. Taylor and P. Pulay. The calculation of abinitio molecular geometries - efficient optimization by natural internal coordinates and empirical correction by offset forces. J. Am. Chem. Soc., 114, 8191 (1992).

[6] J. M. Soler, E. Artacho, J. D. Gale, A. Garcia, J. Junquera, P. Ordejon and D. Sanchez-Portal. The siesta method for ab initio order-n materials simulation. J. Phys.Condens. Mat., 14, 2745 (2002).

[7] P. Ordejon, E. Artacho and J. M. Soler. Self-consistent order-n density-functional calculations for very large systems. Phys. Rev. B, 53, 10441 (1996).

[8] J. P. Perdew, K. Burke and M. Ernzerhof. Generalized gradient approximation made simple. Phys. Rev. Lett., 77, 3865 (1996).

[9] N. Troullier and J. L. Martins. Efficient psudopotentials for plane-wave calculations. Phys. Rev. B, 43, 1993 (1991). 
[10] M. J. Ford, R. C. Hoft and A. McDonagh. First principles theoretical prediction of a new class of self-assembled monolayer: Ethynylbenzenes on gold. J. Phys. Chem. B, 109, 20387 (2005).

[11] J. C. Love, L. A. Estroff, J. K. Kriebel, R. G. Nuzzo and G. M. Whitesides. Selfassembled monolayers of thiolates on metals as a form of nanotechnology. Chem. Rev., 105, 1103 (2005).

[12] Y. Yourdshahyan and A. M. Rappe. Structure and energetics of alkanethiol adsorption on the au(111) surface. J. Chem. Phys., 117, 825 (2002).

[13] A. Bilic, J. R. Reimers and N. S. Hush. The structure, energetics, and nature of the chemical bonding of phenylthiol adsorbed on the $\mathrm{Au}(111)$ surface: Implications for density-functional calculations of molecular-electronic conduction. J. Chem. Phys., 122, 094708 (2005).

[14] Y. Yourdshahyan, H. K. Zhang and A. M. Rappe. N-alkyl thiol head-group interactions with the au(111) surface. Phys. Rev. B, 63, 081405(R) (2001). 


\begin{tabular}{|c|c|c|c|c|c|c|}
\hline \multirow[t]{2}{*}{ Molecule } & \multirow{2}{*}{$\begin{array}{l}\text { No of } \\
\text { atoms }\end{array}$} & \multirow[t]{2}{*}{ Coordinates } & \multirow{2}{*}{$\begin{array}{l}\text { No of } \\
\text { variables }\end{array}$} & \multicolumn{3}{|c|}{ No of CG steps } \\
\hline & & & & I & II & III \\
\hline \multirow[t]{6}{*}{ Water } & \multirow[t]{6}{*}{3} & \multirow[t]{2}{*}{ Cartesian } & 6 & 15 & 15 & 15 \\
\hline & & & 9 & 35 & 37 & 40 \\
\hline & & \multirow[t]{4}{*}{ Z-matrix } & 2 & 6 & 8 & 8 \\
\hline & & & 3 & 3 & 6 & 9 \\
\hline & & & 6 & 3 & 6 & 9 \\
\hline & & & 9 & 4 & 19 & 21 \\
\hline \multirow[t]{7}{*}{ Benzene } & \multirow[t]{7}{*}{12} & \multirow[t]{2}{*}{ Cartesian } & 33 & 25 & 33 & 36 \\
\hline & & & 36 & 7 & 9 & 9 \\
\hline & & \multirow[t]{5}{*}{ Z-matrix } & 2 & 7 & 11 & 18 \\
\hline & & & 11 & 12 & 14 & 20 \\
\hline & & & 30 & 47 & 57 & 69 \\
\hline & & & 33 & 45 & 58 & 63 \\
\hline & & & 36 & 44 & 55 & 66 \\
\hline \multirow[t]{5}{*}{ Hexanedithiol } & \multirow[t]{5}{*}{22} & \multirow[t]{2}{*}{ Cartesian } & 63 & 76 & 108 & 171 \\
\hline & & & 66 & 44 & 46 & 81 \\
\hline & & \multirow[t]{3}{*}{ Z-matrix } & 60 & 20 & 33 & 44 \\
\hline & & & 63 & 24 & 39 & 115 \\
\hline & & & 66 & 32 & 397 & \\
\hline
\end{tabular}

Table 1: Number of conjugate gradient steps required to optimise the geometry of three molecules in Z-matrix and Cartesian coordinates. Columns I, II and III represent progressively stricter convergence criteria for lengths and angles, namely I: (0.04 eV/Å,0.0009 eV/deg); II: (0.02 eV/ $\AA, 0.0004 \mathrm{eV} / \mathrm{deg})$; and III: (0.01 eV/ $\AA, 0.0002$ $\mathrm{eV} / \mathrm{deg}$ ). For the Cartesian coordinate optimisations the angle tolerance is to be ignored. 


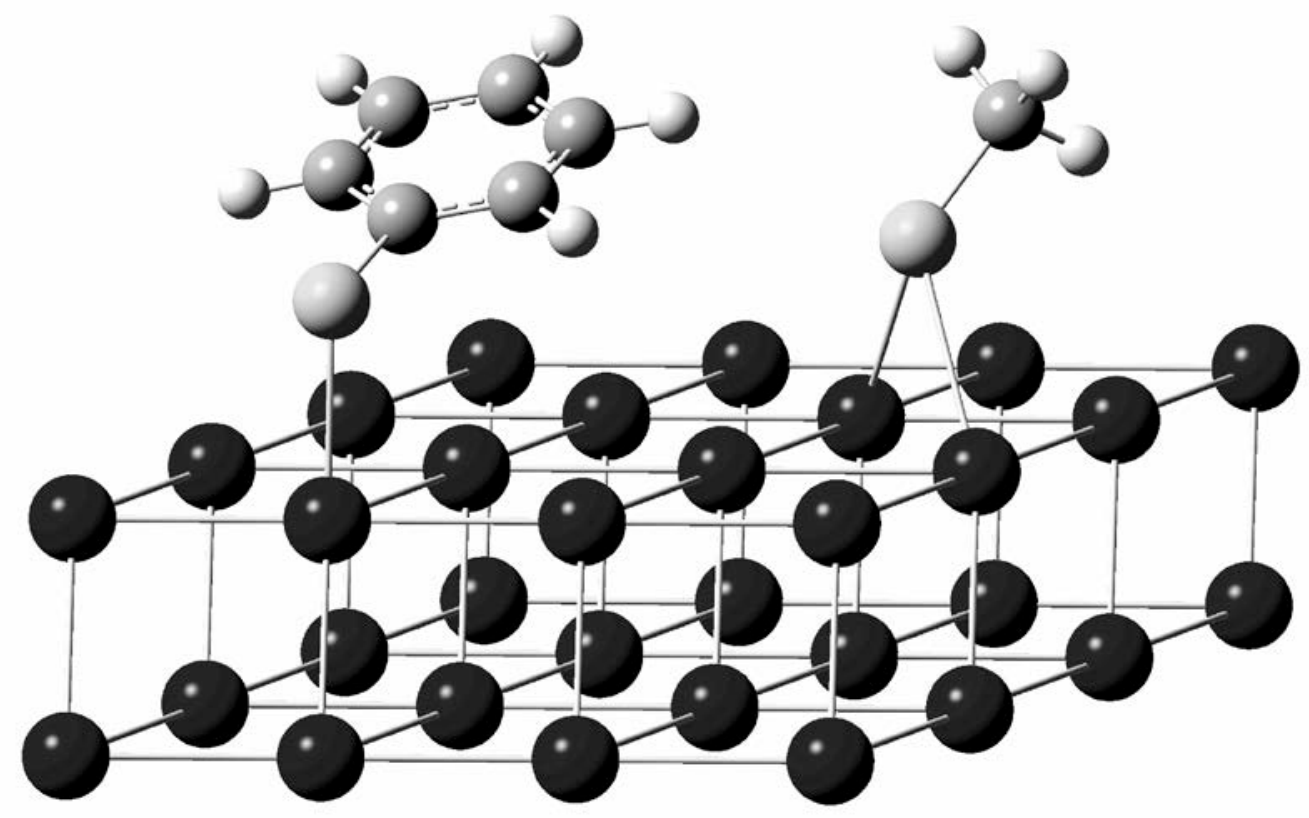

Figure 1. Adsorption of benzenethiol (left) and methanethiol onto the $\mathrm{Au}(111)$ surface. 


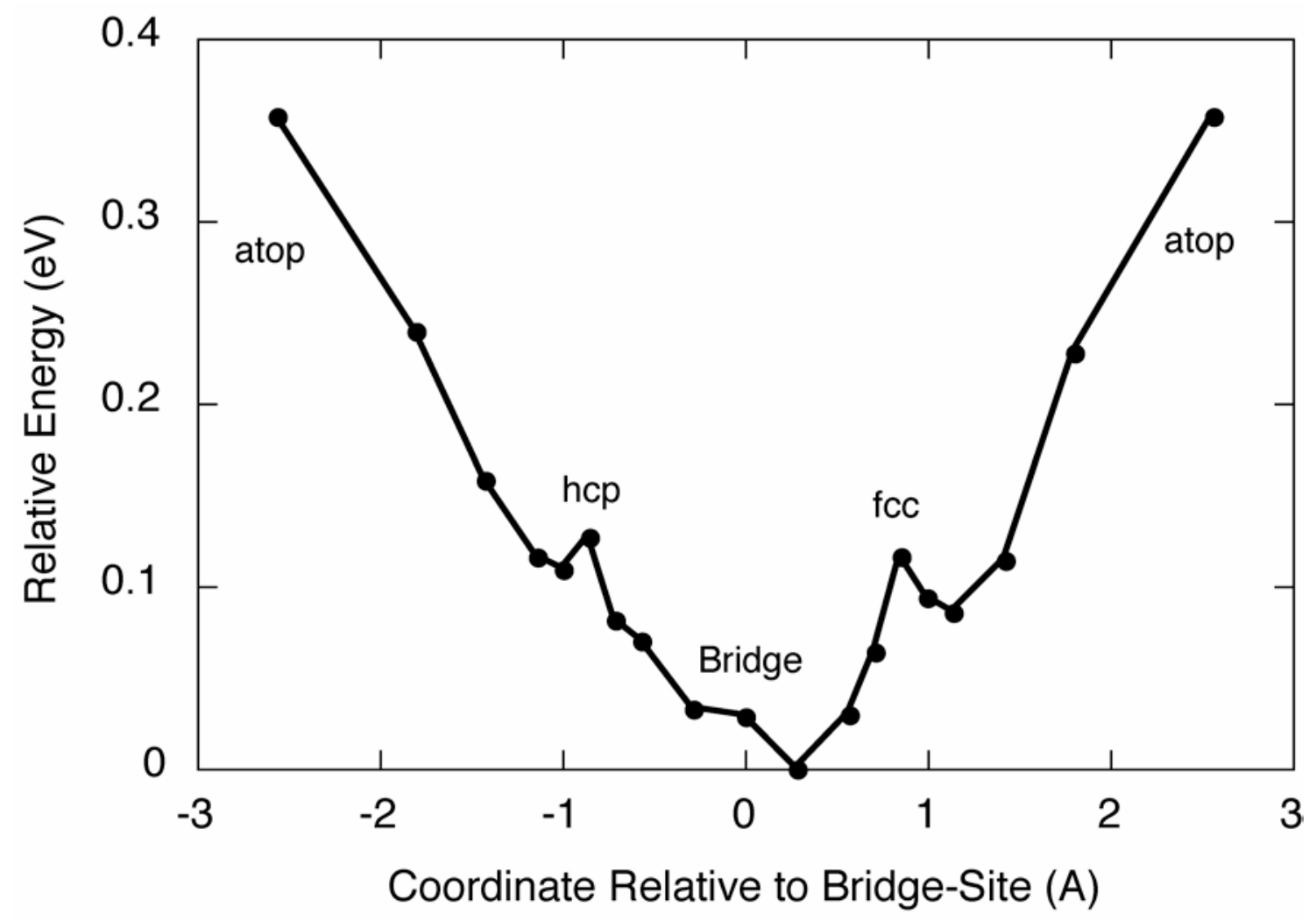

Figure 2. Scan of Potential Energy Surface for Methanethiol moving along the atopbridge-atop path. 